\title{
NOTE: Explaining why the Uranian satellites have equatorial prograde orbits despite the large planetary obliquity.
}

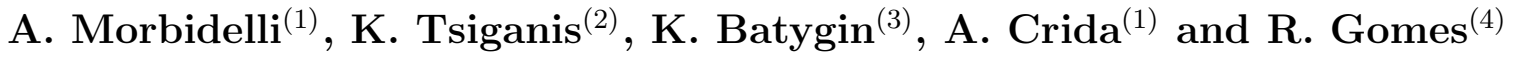 \\ (1) Laboratoire Lagrange, UMR7293, Université de Nice Sophia-Antipolis, CNRS, \\ Observatoire de la Côte d'Azur. Boulevard de l'Observatoire, 06304 Nice Cedex 4, France. \\ (Email: morby@oca.eu / Fax: +33-4-92003118) \\ (2) Department of Physics, Section Astrophysics, Astronomy and Mechanics; Aristotle \\ University of Thessaloniki. Thessaloniki, GR 54124. (E-mail : tsiganis@auth.gr / Fax: \\ $+30-31-995384)$ \\ (3) California Institute of Technology, Division of Geological \& Planetary Sciences; MC \\ 170-25 1200 E. California Blvd., Pasadena, CA 91125. (Email: kbatygin@gps.caltech.edu) \\ (4) Observatório Nacional José Cristino, Rua General Jos Cristino 77, CEP 20921-400, \\ Rio de Janeiro, RJ, Brazil. (Email: rodney@ob.br)
}

Received — 


\begin{abstract}
We show that the existence of prograde equatorial satellites is consistent with a collisional tilting scenario for Uranus. In fact, if the planet was surrounded by a proto-satellite disk at the time of the tilting and a massive ring of material was temporarily placed inside the Roche radius of the planet by the collision, the proto-satellite disk would have started to precess incoherently around the equator of the planet, up to a distance greater than that of Oberon. Collisional damping would then have collapsed it into a thin equatorial disk, from which the satellites eventually formed. The fact that the orbits of the satellites are prograde requires Uranus to have had a non-negligible initial obliquity (comparable to that of Neptune) before it was finally tilted to 98 degrees.
\end{abstract}

\title{
1. Introduction
}

The origin of the large obliquity of Uranus remains elusive. Two scenarios have been proposed: an impulsive tilt due to a collision with a massive body (Safronov, 1966) or a slow tilt due to a resonance between the precession rates of the spin axis and of the orbit (Boué and Laskar, 2010).

A critical constraint, inherent to both of these scenarios, is that the regular satellites of Uranus have essentially equatorial orbits and are prograde relative to the rotation of the planet. Notice that the rotation of the planet is, strictly speaking, retrograde, as Uranus obliquity is about 98 degrees.

In principle, if the satellites were originally coplanar to the equator of Uranus and the planet was tilted slowly (as in Boué and Laskar, 2010), the satellites would have preserved equatorial orbits by adiabatic invariance. Indeed, in the system's current configuration, 
the Laplace plane (the reference plane about which satellite orbits precess) is very close to Uranus' equatorial plane, for all bodies up to Oberon's distance, due to the oblateness of the planet.

In order to tilt Uranus slowly, a resonance between the precession rates of the spin axis and of the orbital plane is required. This means that the former had to be much faster than it is today. In Boué and Laskar (2010) this is achieved by assuming that Uranus originally had a massive satellite with an orbital radius of about 0.01 AU (Satellite X, hereafter). This assumption, however, is problematic because the Laplace plane for Satellite X is close to the orbital plane of Uranus. Thus, Satellite X would not follow the equator during the tilting of the planet and, by virtue of its large mass, would retain the other satellites (particularly Titania and Oberon, as we verified by numerical integration of a slowly tilting system) near its own orbital plane. When Satellite $\mathrm{X}$ is removed by chaotic dynamics, the tilting process is over. Yet, the orbits of the regular satellites of Uranus would remain off equator, as in the impulsive tilting scenario.

In absence of a slow-tilting scenario that does not invoke the existence of Satellite $\mathrm{X}$, we are left with the impulsive tilting scenario as the only viable option. Accordingly, in this Note, we investigate the conditions under which the equatorial, prograde orbits of the regular satellites of Uranus can be reproduced in the context of the collisional tilting scenario.

For clarity, we proceed in steps. We first investigate in Sections $2 \& 3$ the dynamics of a proto-satellite disk around an oblated planet with a tilted spin axis. This highlights the competing effects of the planet's $J_{2}$, the solar perturbation and the self-gravity of the disk. We focus on the conditions that lead the disk to precess incoherently around the planet's equatorial plane and, eventually, to collapse into an equatorial disk. We don't worry at this stage on whether this equatorial disk would be prograde or retrograde. Thus, although we 
fix the obliquity at the current value for Uranus, the dynamics that we study are basically independent on the obliquity value. Then, in Section 4 we consider the fact that Uranus is a retrograde planet (obliquity $\epsilon=98^{\circ}$ ). Thus, a disk originally on the orbital plane of Uranus (as expected if Uranus was tilted from $\epsilon=0^{\circ}$ to $98^{\circ}$ in one shot) would necessarily become an equatorial, retrograde disk. We then investigate which tilting histories of the planet would have a non-negligible probabilities to produce a prograde equatorial disk. In Section 5, we finally discuss the implications of these tilting histories on our understanding of giant planets growth.

\section{Dynamics of a proto-satellite disk around a tilted planet}

It is likely that the giant collision that tilted Uranus occurred during the accretion phase of the planet, when satellites were not yet formed and the planet was surrounded by a tenuous disk of gas, very rich in solids, similar to that usually invoked for the formation of satellites around giant planets (Canup and Ward, 2002). Moreover, even if the planet already had a system of regular satellites at the time of tilting, it is likely that the said

system would have become unstable 1 , developing mutually-crossing orbits. Presumably, the satellites would have then collided with each other, generating a debris disk.

For these reasons, we conduct our investigation assuming for simplicity that, at the time of tilting, the planet was surrounded by a proto-satellite disk of planetesimals. Our nominal disk extends to 1.5 times the distance of Oberon, (i.e. up to $6 \times 10^{-3} \mathrm{AU}$ ), and

\footnotetext{
${ }^{1}$ We checked this with simple $N$-body simulations, which assumed Uranus tilted by 98 degrees and surrounded by a system of regular satellites with the current masses and semi major axes within $1 \%$ of the current ones, but orbits co-planar with Uranus' orbital plane (hereafter denoted for brevity as "the plane of the Sun").
} 
has a mass equal to the combined masses of the five main current regular satellites, (i.e. $10^{-4}$ Uranus masses, $M_{U}$ ). The surface density profile of the disk is assumed to be inversely proportional to the distance from the planet. We assume that, initially, the spin of Uranus is orthogonal to the plane of the Sun and that the disk lays on such a plane.

The dynamical response of such a proto-satellite disk to the impulsive tilting of the planet is far from trivial. Here, we have utilized a simple Laplace-Lagrange-like secular model, where we partition the disk into a system of $N=100$ axisymmetric massive rings that interact with each other gravitationally (see Chapter 7 in Murray and Dermott, 1999). The diagonal terms of the $A$ and $B$ matrices entering the equations (see eq. (7.15) in Murray and Dermott, 1999) are modified to account for the $J_{2}$ term of the potential of the tilted planet, i.e. (see eq. (6.255) in Murray and Dermott, 1999):

$$
A_{j, j}=A_{j, j}+n \frac{3}{2} J_{2}\left(\frac{R_{p}}{a}\right)^{2} \cos \epsilon, \quad B_{j, j}=B_{j, j}-n \frac{3}{2} J_{2}\left(\frac{R_{p}}{a}\right)^{2} \cos \epsilon,
$$

where $R_{p}$ is the radius of Uranus, $a$ is the semi major axis of the ring $j$, and $n$ is its orbital frequency. In the formula above, the usual terms associated to $J_{2}$ are multiplied by $\cos \epsilon$, where $\epsilon$ is the obliquity of Uranus' spin axis, to account for the weakened effect of the oblateness of the planet for large obliquities. We also took into account the scale-height, $h$, of the disk, by using properly softened Laplace coefficients, as in Hahn (2003). The results described below are practically the same for disks with $h=0.01-0.05$

The situation where the planet is suddenly tilted, but the disk initially still lays on the plane of the Sun, is modeled by assuming that the rings initially have $I=\epsilon$ and $\Omega=0^{\circ}$, where $I$ and $\Omega$ are the inclination and longitude of node measured with respect to the equatorial plane of the planet. In addition, all rings are assumed to be initially circular. The Sun is modeled with an additional ring, at a distance of $20 \mathrm{AU}$, also with $I=\epsilon$ and $\Omega=0^{\circ}$. The system is then evolved, according to the Laplace-Lagrange secular equations.

The left panel of Fig 1 shows the longitude of the node of each ring, as a function of 
the ring's distance to Uranus, at four different epochs represented by different colors. The obliquity of Uranus $\epsilon$ is assumed to be $98^{\circ}$. In the inner part of the disk the longitudes of the node are rapidly randomized. This means that the disk loses its disk-like structure and forms a thick torus around the planet. The planetesimals in this portion of the disk can then collide. Collisional break-ups and/or inelastic bouncing would eventually damp the orbital inclination relative to the symmetry plane of the dynamics, i.e. the equatorial plane of the planet. This would form a thin equatorial disk, from which equatorial satellites can eventually accrete. However, Fig 1 shows that this intuitive scenario works only up to a distance of $0.001 \mathrm{AU}$ from the planet, i.e. slightly beyond the orbit of Miranda. Beyond this threshold, the longitudes of the nodes of the rings are not randomized. The rings precess, but their nodal values at any given time trace a relatively smooth function of the distance from the planet, with some small-amplitude oscillations. This means that the disk, under the effect of its own self-gravity, is preserving its disk-like structure, precessing coherently around the planet's equator.

More precisely, close to the 0.001 AU threshold, the disk is significantly warped (i.e. the values of $\Omega$ change significantly with the distance from Uranus), but beyond $\sim 0.003 \mathrm{AU}$ the disk is characterized by almost rigid precession (i.e. all values of $\Omega$ are about the same). The inclinations (not shown in the plot) of the rings in the region where the disk precesses rigidly remain practically constant over the precession of $\Omega$. However, in the warped region, they show regular oscillations with non-negligible amplitude, related to the precession of $\Omega$. Thus, at any given time, the inclinations change smoothly with $\Omega$ and the distance from the planet.

Two caveats about this calculation needs to be discussed. First, the validity of the Laplace-Lagrange equations for this problem may appear doubtful, because the inclinations $I$ of the rings are large, whereas the equations have been developed asymptotically for 

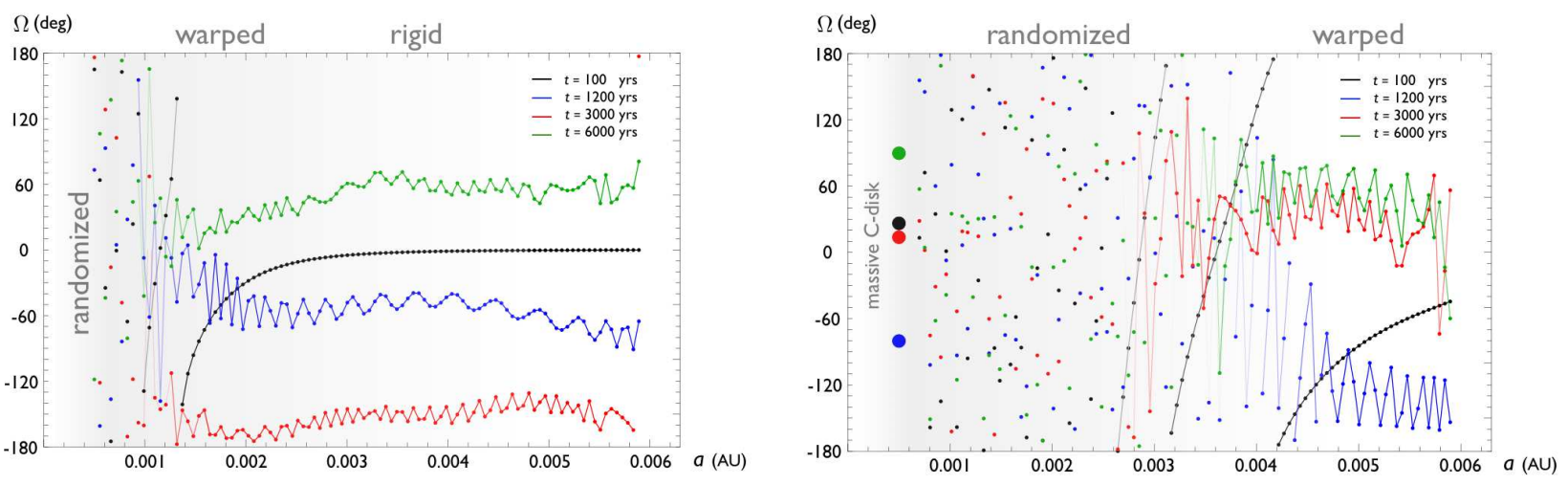

Fig. 1. - Longitudes of the nodes of the concentric rings that compose the proto-satellite disk in our Lagrange-Laplace model, as a function of the the rings' distance to the planet. Diffent colors represent different times, as labelled. The regions where the nodes are randomized, the disk is warped or precesses quasi-rigidly are also labelled. Left panel: the nominal case, with Uranus current $J_{2}$. Right panel: the case where a transient equatorial collisional disk of $0.01 M_{U}$ is present at $\sim 3$ Uranian radii. The node of this disk is represented by a filled dot.

systems with $e, I$ that tend to 0 . In reality, the inclinations are large relative to the planet's equator, and this fact is taken into account by multiplying the coefficients in (1) by $\cos \epsilon$. But the relative inclinations of the rings are originally very small, in accordance with the Laplace-Lagrange theory. Thus, the equations are valid and will remain valid as long as the relative inclinations of the rings remain small, which is for all times in the portion of the disk that precesses coherently, beyond 0.001 AU. The Lagrange-Laplace approximation breaks down in the inner part of the disk, once the nodes get randomized. However, this is enough for our purpose, which is just to investigate whether node randomization takes place and where.

The second caveat concerns our assumption of an initial circular and coplanar disk. In fact, Parisi and Brunini (1997) showed that the impulsive tilting of Uranus implies an impulsive change in the orbital velocity of the planet of about $2 \mathrm{~km} / \mathrm{s}$. As a result, satellites or disk particles originally beyond 70-90 Uranus radii $\left(1.2-1.5 \times 10^{-2} \mathrm{AU}\right)$ would be 
removed from the system. The disk that we consider is much smaller than this threshold. Nevertheless, disk particles would get eccentric and/or inclined relative to the new orbital plane of the planet, the magnitude of $e$ and $i$ growing as $\sqrt{a}$. However, the trajectories of the disk particles would start to intersect with each other. This would lead to a rapid collisional damping, until the disk recovers its circular and coplanar structure. For this reason, we think that our initial conditions (a circular and coplanar disk) are nevertheless appropriate for our goal, i.e. determining the distance from the planet within which the orbital planes of the disk particles are randomized.

The conclusion that we draw from Fig. 1 is that the precession of the disk relative to the equator alone cannot explain the equatorial orbits of the satellites beyond Miranda. In fact, a disk precessing coherently, as that illustrated in the left panel of Fig 1 beyond 0.001 AU, would eventually form satellites on its own plane, i.e. on highly-inclined orbits relative to the equator of the planet.

Note that our nominal disk represents a best-case scenario for the randomization of the nodes. Had we chosen a disk less radially extended (up to $4 \times 10^{-3}$ AU, for instance) or more massive, the effect of self-gravity would have been even more important and the region where the nodes randomize would have been confined closer to the planet. This strengthens the conclusion presented above. This implies that the equatorial configuration of the Uranian satellites requires a more complex explanation.

\section{An enhanced $J_{2}$ for the just-tilted Uranus}

The results from the previous section highlight the need for a dynamical mechanism that can broaden the region where the nodes of the disk particles get randomized, because only in this region the disk loses coherence and can collapse on the planet's equatorial plane. 
Recall that the disk loses coherence at a distance from the planet where the precession rate around the equator forced by the $J_{2}$ term dominates the precession rate around the disk's mid-plane that is forced by the disk's self-gravity. Because we cannot reduce the self-gravity of the proto-satellite disk, the only option is to find a mechanism that enhances Uranus' $J_{2}$ in the aftermath of the collision.

A first idea that we explored is that the inner part of the disk, within $0.001 \mathrm{AU}$ damps on the equator and forms an equatorial satellite (with a mass comparable to that of Miranda). This enhances the effective $J_{2}$ of the planet felt by the outer portion of the disk, thus extending the zone in which the disk precesses incoherently, leading to the collapse of another ring on the equator, etc. However, we found that this mass is too small, and this effect is negligible.

We then turned to published simulations of the collisional tilting scenario for Uranus. These simulations (Slattery et al., 1992) show that the impact should have generated an equatorial disk of debris, accounting for $\approx 1$ to $3 \%$ of the mass of Uranus, namely about 100 times more massive than the proto-satellite disk, but mostly confined within 3 Uranian radii. We call this disk the C-disk, equivalent to the proto-Lunar disk, as it was generated in the collision, in order to avoid confusion with the proto-satellite disk considered up to now.

The C-disk could not generate the current regular satellites of Uranus, because the latter are too far away (Canup and Ward, 2000). In fact, most likely as the C-disk spread outside of the Roche lobe of the planet, it formed satellites (Charnoz et al., 2010) which, being situated inside the corotation radius, tidally migrated onto the planet. The existence of this massive C-disk (or of the close satellites that it generated), however, is equivalent to increasing the planet's $J_{2}$ enormously.

The right panel of Fig 1 shows the result of our Laplace-Lagrange model of the 
evolution of the proto-satellite disk, when we account for a C-disk of $0.01 M_{U}$. Again, the obliquity of Uranus is $\epsilon=98^{\circ}$. The C-disk is represented by a circular ring at 3 Uranian radii with $I=0$, but with a mass multiplied by a factor $\cos \epsilon$ to account for its inclination $\epsilon$ relative to the proto-satellite disk, as we did for the $J_{2}$ coefficients in (1). As one can see from the plot, the region where the longitude of nodes in the proto-satellite disk are randomized now extends to $0.003 \mathrm{AU}$ (almost the distance of Oberon). The radial extent of the randomized disk can be enhanced further by assuming a more massive C-disk. For example, if we take the mass of the C-disk to be $0.03 M_{U}$ the randomized region extends to $0.0045 \mathrm{AU}$ and so on.

We have checked our results with N-body simulations that take the self-gravity of the proto-satellite disk into account. The C-disk is modeled with a single massive satellite on a circular orbit at 3 Uranian radii on the equatorial plane of the planet. The proto-satellite disk is modeled with 2,000-8,000 equal-mass particles, initially on circular orbits on the plane of the Sun. All particles interact with each other, with a smoothing length equal to 0.5-3 Hill spheres of the innermost particle, which is a equivalent to a few times the particle radius. We also tested disks twice as massive as our nominal case and with different initial scale heights. We performed several simulations and found our results to be robust with respect to changes in the values of the above parameters. The calculations were performed using a version of SyMBA (Duncan et al., 1998), suitably modified so that the $N^{2}$ force calculation is performed on a GPU card, with the help of the SAPPORO library (Gaburov et al., 2009). The time-step was chosen equal to $1 / 30$ times the period of the massive satellite.

The results of two simulations (4,000 particles, nominal mass) are shown in Fig. 2, In the case where the C-disk has a mass of $0.01 M_{U}$ (left panel), after a time of $1000 \mathrm{yr}$, the randomized region extends to $0.003 \mathrm{AU}$, in excellent agreement with our analytic 

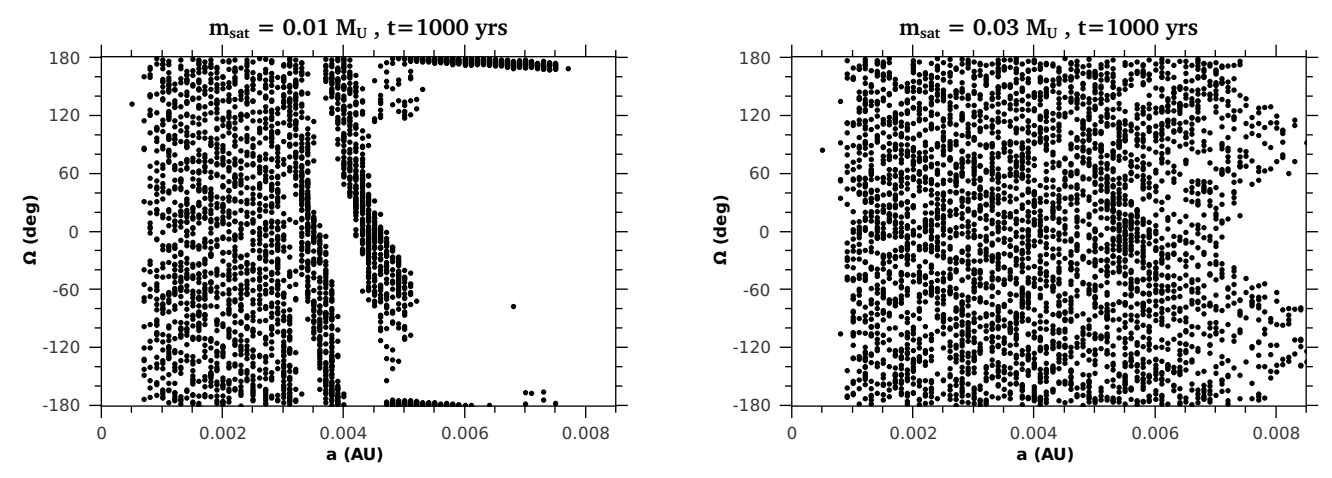

Fig. 2.- Longitudes of the nodes of the disk particles as a function of their semi major axis, after $1000 \mathrm{y}$ years of numerical simulation. The left panel includes the effect of a $0.01 M_{U}$ satellite at 3 Uranian radii. The right panel is for a $0.03 M_{U}$ satellite.

model. Beyond this threshold, though, the disk is extremely warped, up to about 0.005 AU (i.e. beyond the current position of Oberon). By mutual interactions, the particles in the 0.003-0.005 AU region also become quite eccentric and cross each other (the Laplace-Lagrange approximation is not valid there). On the longer run, therefore, the randomization is expected to extend to $0.005 \mathrm{AU}$. Instead, in the case where the C-disk has a mass of $0.03 M_{U}$ (right panel), the nodes of the disk particles are randomized up to $0.006 \mathrm{AU}$ within the same timescale.

We conclude from these experiment that, if the collision that tilted Uranus really formed a massive, equatorial transient disk, as in the simulations of Slattery et al. (1992), the proto-satellite disk would have lost coherence up to a distance at least equal to that of Oberon, dispersing symmetrically around the equator of the planet. Collisional damping would have then forced the proto-satellite disk to collapse onto the equatorial plane, where the observed satellites, from Miranda to Oberon, could form. It is likely that, during this process, some of the disk's mass would be "lost", instead of accreting on the satellites. However, our simulations give practically the same results even for a disk twice as massive 


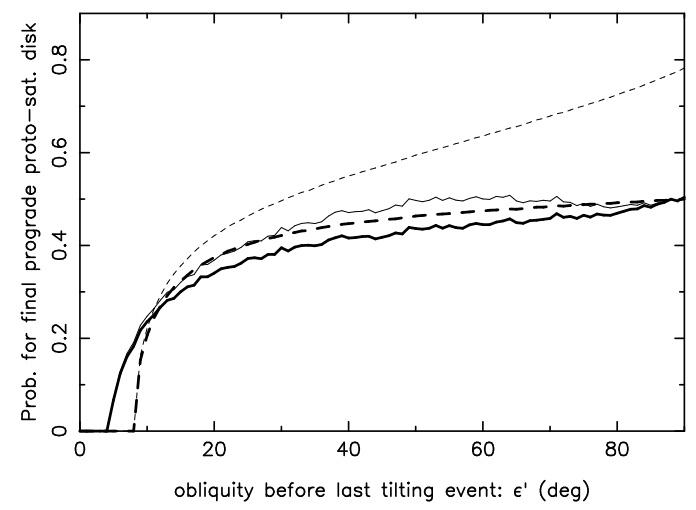

Fig. 3.- The probability that the proto-satellite disk has a prograde orientation relative to the final spin axis of Uranus as a function of the obliquity $\epsilon^{\prime}$ that Uranus had prior to the final tilting. The results of four calculations are presented according to the assumptions explained in the text.

as the combined mass of the current satellite system. This is because the mass ratio between the C-disk and the proto-satellite disk is of $\sim 100$.

\section{Why are Uranus satellites prograde?}

If Uranus had been tilted abruptly from an obliquity of 0 to one of 98 degrees, the mechanism described in the previous section would have produced a system of equatorial, but retrograde satellites. This is because the precession of the particles of the proto-satellite disk around the equator and their collisional damping would preserve the total angular momentum of the disk relative to the spin axis of the planet, which is negative from the very beginning (i.e. at the time when the planet is tilted and the disk is still on the plane of the Sun).

To have the disk collapse by collisional damping onto an equatorial, prograde disk, it is necessary that the obliquity of Uranus was not null when the planet's final tilting episode occurred. Let's call $\epsilon^{\prime}$ this pre-final-tilt obliquity. 
We have computed, with Monte-Carlo simulations, the probability that the final proto-satellite disk is prograde as a function of $\epsilon^{\prime}$. We have done four simulations, adopting different assumptions; in each simulation $\epsilon^{\prime}$ progresses from $0^{\circ}$ to $90^{\circ}$ by one-degree steps. First, we assume that, when the planet had an obliquity $\epsilon^{\prime}$, it was surrounded by a proto-satellite disk that precessed rigidly around its equator with an inclination $I=\epsilon^{\prime}$. From the results in the previous sections, this is expected if such obliquity had been acquired impulsively from an initial obliquity of 0 . The Monte Carlo calculation in this case assumes two random angles: (i) the azimuthal orientation of the final spin vector (of obliquity $\epsilon=98^{\circ}$ ) relative to the plane of the Sun and (ii) the precession phase of the proto-satellite disk at the time of the final tilting. The result is illustrated with the bold solid curve in Fig. 3. Second, we assume that, when the planet had an obliquity $\epsilon^{\prime}$, its proto-satellite disk had the time and the conditions to become equatorial. In this case, the Monte Carlo calculation has to assume just one random angle (the orientation of the new vs. the old spin vectors). The result is illustrated with a bold dashed curve in Fig. 3 , Then, we relax the assumption that the relative orientations of the original and final spin vectors of the planet are equi-probable. Instead, we assume that probability that the spin vector has a change $\overrightarrow{\delta \epsilon}=\vec{\epsilon}-\overrightarrow{\epsilon^{\prime}}$ is proportional to $1 /\|\overrightarrow{\delta \epsilon}\|$ (here $\overrightarrow{\epsilon^{\prime}}$ and $\vec{\epsilon}$ are, respectively, the unitary spin vectors of the planet before and after the final tilting and $\|$.$\| denotes the$ Euclidean norm of a vector). If the disk is precessing around the planetary equator before the final tilt, the result is illustrated by a thin solid curve and it is not very different from that obtained in the first calculation (compare with the bold solid curve). This is because the scalar product between the angular momentum vectors of the disk and the planet is more sensitive to the precession phase of the disk than to the relative orientation of the initial and final spin vectors of the planet. Instead, if the disk was equatorial, the result is illustrated by the thin dash curve and is significantly different from that obtained in the second calculation, for large values of $\epsilon^{\prime}$ (compare with the the dash bold curve). 
As one can see in Fig. 3, the probability to have a prograde disk is null if $\epsilon^{\prime}$ is smaller than 4 or 8 degrees for a rigid-precessing disk or an equatorial disk, respectively, for obvious geometric reasons. However, in all cases we find that the probability of ending up with a prograde satellite system increases rapidly with $\epsilon^{\prime}$ and, for $\epsilon^{\prime}=30^{\circ}$ (like the one of Neptune), it exceeds $40 \%$.

\section{Conclusions}

We conclude that the collisional tilting scenario for Uranus is consistent with the prograde, equatorial character of the orbits of its regular satellites, as well as the size of the system. The fact that the satellites are prograde, implies that Uranus was not tilted from 0 to 98 degrees in one shot. Instead, it had to have had a non-negligible obliquity, prior to the final giant impact. Thus, Uranus should have experienced at least two giant collisions.

This result, together with the obliquity of Neptune, which also has no other explanation than a collisional tilt, suggests that giant impacts, affecting the obliquities, were rather common during the growth of the ice-giants of the solar system. Thus, these planets presumably did not grow by the sole accretion of small planetesimals as often envisioned. Instead, towards the end of their accretion history, they should have experienced a phase similar to that characterising the process of terrestrial planet formation, i.e. dominated by giant impacts with other large planetary embryos. Past and future models of growth of giant planet cores should be confronted with this constraint.

\section{References}

- Boué, G., Laskar, J. 2010. A Collisionless Scenario for Uranus Tilting. The Astrophysical Journal 712, L44-L47. 
- Canup, R. M., Ward, W. R. 2000. A Possible Impact Origin of the Uranian Satellite System. Bulletin of the American Astronomical Society 32, 1105.

- Canup, R. M., Ward, W. R. 2002. Formation of the Galilean Satellites: Conditions of Accretion. The Astronomical Journal 124, 3404-3423.

- Charnoz, S., Salmon, J., Crida, A. 2010. The recent formation of Saturn's moonlets from viscous spreading of the main rings. Nature 465, 752-754.

- Duncan, M. J., Levison, H. F., Lee, M. H. 1998. A Multiple Time Step Symplectic Algorithm for Integrating Close Encounters. The Astronomical Journal 116, 2067-2077.

- Gaburov, E., Harfst, S., Portegies Zwart, S. 2009. SAPPORO: A way to turn your graphics cards into a GRAPE-6. New Astronomy 14, 630-637.

- Hahn, J. M. 2003. The Secular Evolution of the Primordial Kuiper Belt. The Astrophysical Journal 595, 531-549.

- Murray, C.D. and Dermott, S.F. 1999. Solar System Dynamics. Cambridge University Press.

- Parisi, M. G., Brunini, A. 1997. Constraints to Uranus' Great Collision-II. Planetary and Space Science 45, 181-187.

- Safronov, V. S. 1966. Sizes of the largest bodies falling onto the planets during their formation. Sov. Astron., 9, 987-991.

- Slattery, W. L., Benz, W., Cameron, A. G. W. 1992. Giant impacts on a primitive Uranus. Icarus 99, 167-174. 